\title{
Conduction in rectangular quasi-one-dimensional and two-dimensional random resistor networks away from the percolation threshold
}

\author{
Thomas Kiefer, ${ }^{*}$ Guillermo Villanueva, ${ }^{\dagger}$ and Jürgen Brugger ${ }^{\ddagger}$ \\ Microsystems Laboratory, École Polytéchnique Fédérale de Lausanne (EPFL), Lausanne 1015, Switzerland
}

(Received 19 February 2009; revised manuscript received 7 July 2009; published 11 August 2009)

\begin{abstract}
In this study we investigate electrical conduction in finite rectangular random resistor networks in quasione and two dimensions far away from the percolation threshold $p_{c}$ by the use of a bond percolation model. Various topologies such as parallel linear chains in one dimension, as well as square and triangular lattices in two dimensions, are compared as a function of the geometrical aspect ratio. In particular we propose a linear approximation for conduction in two-dimensional systems far from $p_{c}$, which is useful for engineering purposes. We find that the same scaling function, which can be used for finite-size scaling of percolation thresholds, also applies to describe conduction away from $p_{c}$. This is in contrast to the quasi-one-dimensional case, which is highly nonlinear. The qualitative analysis of the range within which the linear approximation is legitimate is given. A brief link to real applications is made by taking into account a statistical distribution of the resistors in the network. Our results are of potential interest in fields such as nanostructured or composite materials and sensing applications.
\end{abstract}

DOI: 10.1103/PhysRevE.80.021104

PACS number(s): 64.60.ah, 64.60.an, 46.65.+g, 05.60.Cd

\section{INTRODUCTION}

The knowledge about the physical properties of composite, porous, or discontinuous materials exhibiting critical phenomena or phase transitions [1-3] has great importance in many fields of science and engineering such as functional polymer materials [4] or chemical sensing applications $[5,6]$. Characteristic properties such as critical thresholds, conduction, or cluster sizes can be described by percolation theory, which has been subject of many studies over the last decades [7-9]. Its capability to predict transport processes in the vicinity or away from the critical region makes it applicable to a variety of physical phenomena such as water flow through porous media [10], diffusion in disordered media [11] or electrical conduction in discontinuous materials [5].

In the simplest case a percolation system consists of connected bonds (bond percolation), which are randomly placed on a lattice in $n$ spacial dimensions with probability $p$. In the limit of an infinite system a set of connected bonds appears at a critical fraction $p_{c}$ of occupied bonds (the percolation threshold), which spans across the entire system. The properties of this so-called infinite cluster, such as size or conductivity, are described as a function of $p-p_{c}$. In the vicinity of the percolation threshold, which is a sharp transition, power laws with specific exponents apply, which are of the generic form $x(p) \sim\left(p-p_{c}\right)^{y}[8]$.

In real systems, finite-size effects and related boundary effects become non-negligible and geometrical and/or topological constraints might come into play $[6,12,13]$. Finite size scaling techniques $[8,14]$, renormalization group theory [8,15-17], and common Monte Carlo techniques [8] have been used to address such issues.

Conduction in square systems has been extensively studied [7], especially in the critical region around $p_{c}$. Conduc-

\footnotetext{
*thomas.kiefer@epfl.ch

† guillermo.villanueva@epfl.ch

¥juergen.brugger@epfl.ch
}

tion phenomena can be studied using random resistor networks, which are the electrical embodiment of a percolation system [7]. Here, the conduction $G$ growths nonlinearly with $p$ following a power law with a critical exponent $t(t \approx 1.31$ in two dimensions [18]) and obeys finite-size scaling $[18,19]$. In the region far away from $p_{c}$, effective medium theory [7] gives a linear approximation for $G(p)$, where $G(P) \sim p-p_{c}$ with $p_{c}=2 / z$ and $z$ as the coordination number (the number of next neighbors) of the undiluted lattice, where $p=1$. A combination of real-space normalization and effective medium theory showed a quite good approximation to the conduction problem over the entire range of $p$ [16].

Most existing literature refers to systems with a square geometry in the infinite limit, yet only few studies exist on percolation in systems with finite rectangular network geometries. It has been shown that geometrical constraints strongly affect the percolation properties, making it interesting for the structuring and design of materials for, e.g., sensing applications [6]. For the first time, Monetti and Albano [20-22] studied percolation cluster properties by Monte Carlo techniques and finite-size scaling on rectangular percolation systems. Tsubakihara [23] found the percolation probabilities for site percolation in two dimensions based on a modified finite-size scaling function. Masihi et al. [24] proposed a new scaling function for percolation thresholds in systems of moderate geometrical aspect ratios and showed finite-size scaling for the connectivity (fraction of connected sites) and its variation in site percolation.

While effective medium theory holds well for conduction away from the percolation threshold on square systems, no model exists yet for finite systems with rectangular geometries to our knowledge. In this paper, we investigate conduction far away from the percolation threshold for various topologies: a quasi-one-dimensional (1D) and the square and triangular two-dimensional (2D) systems. The choice of those topologies is motivated by their occurrence and applicability to nanostructured or functional materials such as arrays of electrodeposited metallic mesowires on highly orien- 


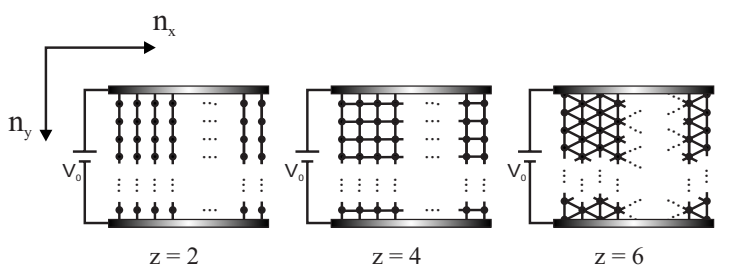

FIG. 1. Schematic representation (not to scale) of resistor networks with various coordination numbers $z$ : quasi-one-dimensional system (parallel linear chains) with $z=2$, two-dimensional square $(z=4)$, and triangular lattice $(z=6)$. Lines represent resistors; dots represent nodes. The ends are connected by isopotential bar and an electric potential $V_{0}$ is applied across.

tated pyrolytic graphite surfaces [25], electromigration failure in metallic wire networks [26] or conducting polymers [27] to give just a few examples. Due to their applicability to, e.g., sensors even above the percolation threshold, the modeling of electrical conduction in this regime is of practical interest.

In contrast to the quasi-one-dimensional system, which shows pronounced nonlinear characteristics and a strong dependence on the geometry, a linear approximation for conduction in two-dimensional bond percolation based on Monte Carlo simulations and previous work on connectivity in site percolation can be formulated. The geometrical effects are less pronounced in two dimensions. We further give a rough estimate of the range in which this approximation in $2 \mathrm{D}$ is legitimate. In the last section we briefly make a link from our percolation model to physical quantities as it is the case in, e.g., sensors, where the fraction of bonds is only indirectly accessible.

\section{SIMULATION MODEL}

In our model, we consider a bond percolation lattice where each bond is placed with an independent probability $p$ and left void with probability $q=1-p$. Replacing the bonds by unit resistors/conductances turns the system into a random resistor network [7]. An electric unit potential $V_{0}$ is applied at two opposing ends of the network, which are connected by isopotential bars. We define a longitudinal axis $y$ into the direction of the electric field where $n_{y}$ is the number of resistors in this direction, and a transversal axis $x$ with $n_{x}$ resistors and open boundary conditions, which is perpendicular to it (see schematic in Fig. 1). Using Kirchhoff's voltage law for each loop in the circuit, a set of linear equations is obtained [7]

$$
\sum_{j} V_{j}=g_{i j}^{-1} I_{i}=0
$$

where $g_{i j}$ are randomly chosen conductances at adjacent sites $i$ and $j$, and $I_{i}$ is the current through the $i$ th conductance of the network. The mean conductance $G(p)$ of the resulting resistor network can be calculated by generating a number of different realizations (in our case 1000) for constant $p$ and solving the set of equations by, e.g., a conjugate gradient method.

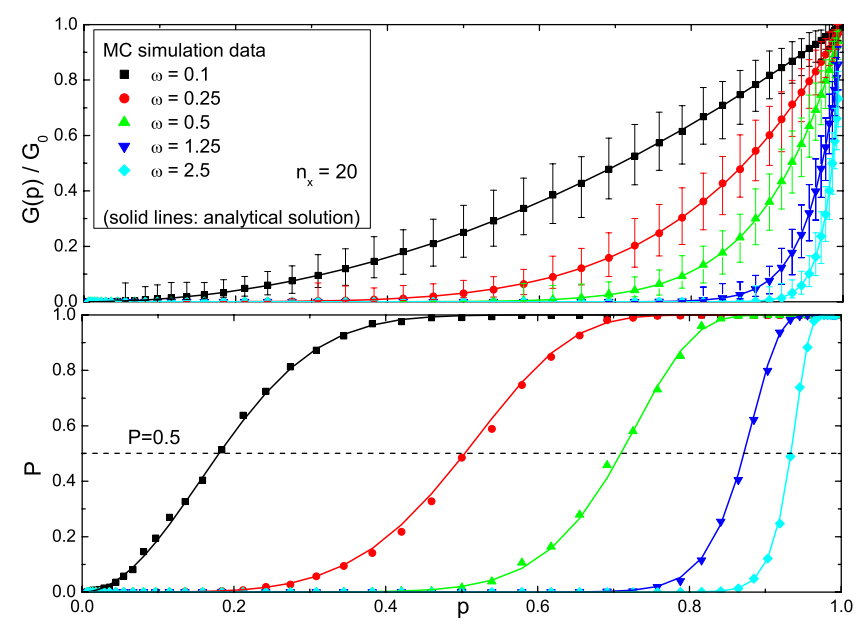

FIG. 2. (Color online) Normalized conduction and percolation probability for a quasi-one-dimensional system with $n_{x}=20$ and various aspect ratios $\omega$. Data points represent Monte Carlo simulation data with standard deviations; the solid lines represent the analytical solutions. Apparent percolation thresholds are defined where $P=0.5$. Symmetry of $P$ around $P=0.5$ breaks up toward the boundaries $p=0$ and $p=1$.

The fraction of network realizations, which leads to a cluster that connects across one direction, is called the percolation probability $P$. In the infinite limit there is a sharp transition where $P=0$ for $p<p_{c}$ and $P=1$ for $p \geq p_{c}$. For finite systems this transition smears out and $P$ becomes a monotonously increasing function of $p$. In this case another definition must be used. In two-dimensional site percolation, Masihi et al. [24] introduced an apparent percolation threshold $\tilde{p}_{c}$ where the probability to find a percolating cluster is $P=0.5$. A change in the geometrical aspect ratio $\omega=n_{y} / n_{x}$ of the network causes a shift in $\widetilde{p}_{c}$. With their definitions, this shift was found to be symmetric around the isometric case, which is the case where the aspect ratio $\omega=1$. The introduction of a new scaling function, which will be used in Sec. IV, allowed the use of regular finite-size scaling to describe the mean connectivity and its standard deviation.

Using similar definitions, we performed Monte Carlo simulations on random resistor networks with quasi-onedimensional as well as two-dimensional square and triangular lattices under moderate geometrical aspect ratios. A schematic drawing is shown in Fig. 1.

\section{QUASI-ONE-DIMENSIONAL SYSTEM}

Linear chains of resistors, which are arranged in parallel, can be considered as a quasi-one-dimensional system since each of the chains can be independently treated as onedimensional. Unlike for a single linear chain, the percolation probability $P$ in a finite system of parallel chains is not a sharp transition anymore. The definition of an apparent percolation threshold is therefore required. Monte Carlo simulations indicate that $P$ is approximately symmetric around $P=0.5$ but this symmetry breaks up when $\mathrm{p}$ approaches the boundaries $p=0$ and $p=1$ (see Fig. 2). Changing the geometrical aspect ratio $\omega$ causes significant shifts of $P$ over a 


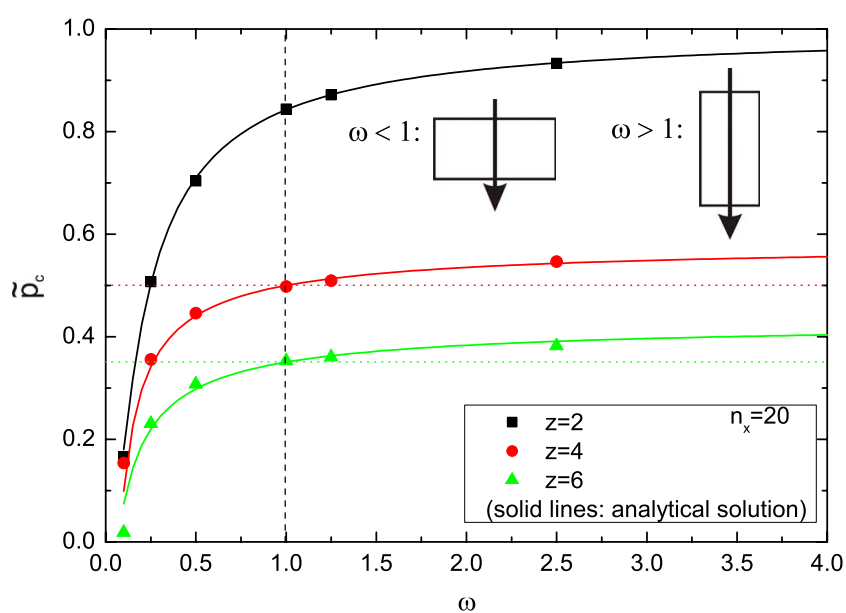

FIG. 3. (Color online) Monte Carlo simulation results and analytical model for apparent percolation thresholds for various coordination numbers $z$ as a function of aspect ratio $\omega$. The isotropic case $\omega=1$ is marked by the vertical dashed line. Horizontal dashed lines represent corresponding percolation thresholds of the infinite systems. For $z=2$ (quasi-1D) this is $p_{c}^{\infty}=1$.

wide range of $p$. In good approximation, we define can the apparent percolation threshold $\widetilde{p}_{c}$ where $P=0.5$ [9]. This definition coincides with the definition in $2 \mathrm{D}$, yet is has to be pointed out that no correlation exists between the one- and two-dimensional case.

An analytical solution to this problem is readily obtained by using basic probability theory: given the probability for a single 1D chain to conduct, which is $P=p^{n_{y}}$ [9], the percolation probability $P$ to find at least one conductive chain can be calculated by the complementary probability of having no single conductive chain, which yields

$$
P=1-\left(1-p^{n_{y}}\right)^{n_{x}},
$$

with the $50 \%$ criterion one obtains $P_{50 \%}=0.5=1-\left(1-\widetilde{p}^{n_{y}}\right)^{n_{x}}$, where $\widetilde{p}_{c}$ is the apparent percolation threshold, and Eq. (2) yields

$$
\tilde{p}_{c}^{1 D}=\left(1-0.5^{n_{x}^{-1}}\right)^{n_{y}^{-1}},
$$

where $n_{x}$ is the number of chains and $n_{y}$ the number of resistors per chain. In the infinite limit $\widetilde{p}_{c}^{1 D}$ converges to 1 . This analytical solution and the values extracted from numerically obtained data show good agreement as it can be seen in Fig. 2 for $P$ and Fig. 3 for $\tilde{p}_{c}^{1 D}$. The apparent percolation thresholds cover approximately $80 \%$ of the range in between the boundaries $p=0$ and $p=1$ for aspect ratios $\omega<1$ and the given system size of $n_{x}=20$. Larger systems will result in higher percolation thresholds for a given aspect ratio.

The conduction for a parallel set of linear chains can simply be derived from the probability of conduction for a single chain, multiplied by the number of chains and yields

$$
G(p)=p^{n_{y}} n_{y}^{-1} n_{x}=p^{n_{y}} G_{0},
$$

where $G_{0}=n_{x} / n_{y}$ is the conduction of the undiluted lattice. Figure 2 shows that the model fits well the simulated data over the entire range of $p$. The conduction $G$ as a function of $p$ is highly nonlinear and, such as the percolation probability, changes drastically with the aspect ratio $\omega$.

\section{TWO-DIMENSIONAL SQUARE AND TRIANGULAR LATTICE}

In two-dimensional rectangular percolation systems, no simple analytical expression for the conduction can be derived from probability theory. In a first step we will confirm that the scaling function for percolation thresholds introduced by Masihi et al. [24] applies to our bond percolation model. In analogy, we use a scaling function, which has been (as an approximation) defined as

$$
\Lambda(\omega)=c\left(\omega^{1 / \nu}-1\right),
$$

where $\omega$ is the aspect ratio of the system, $\nu$ the correlation length exponent $(\nu=4 / 3)$ and $c$ a constant factor. This function in two dimensions is symmetric with respect to the longitudinal and transversal direction of the lattice with $\Lambda_{x}(\omega)$ $=-\Lambda_{y}(\omega)$. With this, the apparent percolation thresholds scale with the system size as

$$
\widetilde{p}_{c}^{2 D}=p_{c}^{\infty}+\Lambda(\omega) n_{y}^{-1 / \nu},
$$

where $p_{c}^{\infty}$ is the percolation threshold of the infinite system [24]. The constant $c$ can be determined as follows: First, numerical values for $\widetilde{p}_{c}$ are obtained by Monte Carlo simulations of lattices with various aspect ratios. Using Eq. (6), corresponding values for the scaling function are obtained and plotted against $\omega^{1 / \nu}-1$ by using Eq. (5). The slope of the resulting linear plot is equal to $c$. For bond percolation in two dimensions we find $c=0.82 \pm 0.04$ for both $z=4$ and $z=6$ and is therewith independent of the topology. Note that the variation of \pm 0.04 adds an uncertainty factor of approximately $5 \%$ to the prediction of the model.

Figure 3 shows simulation data and calculated values for apparent percolation thresholds as a function of aspect ratio for various topologies and $n_{x}=20$. Dots indicate data points; solid lines indicate solutions of the analytical model. The calculated values show slight deviations compared with the data of the Monte Carlo simulations but still lie within the range of uncertainty, which is given by the constant $c$. The relative deviations, averaged over three different system sizes $n_{x}=10,20$, and 50 , were $0.7 \% \pm 1.3 \%$ for $z=2$, $2.4 \% \pm 2.5 \%$ for $z=4$, and $3.3 \% \pm 1.9 \%$ for $z=6$, with decreasing values for larger systems and larger errors for smaller aspect ratios $\omega$. For $\omega=1$ the apparent percolation thresholds in two dimensions coincide with the percolation thresholds of an infinite system as expected.

Let us now focus on the conduction problem in 2D. In the infinite limit a rectangular system with a finite aspect ratio is not affected by its boundaries and behaves like a square system. The apparent percolation threshold becomes the percolation threshold of the infinite square system. In analogy, the slope of the normalized conduction $d\left(G / G_{0}\right) / d p$ far away from $p_{c}$ converges in good approximation to the slope at infinity, which will here be referred to as $m^{\infty}$. For $z=4$ one obtains $m^{\infty}=2$ and for $z=6, m^{\infty} \approx 1.532$. Due to this analogy we assume the conduction in the linear regime to follow a similar scaling function with the same parameter $c$. We de- 


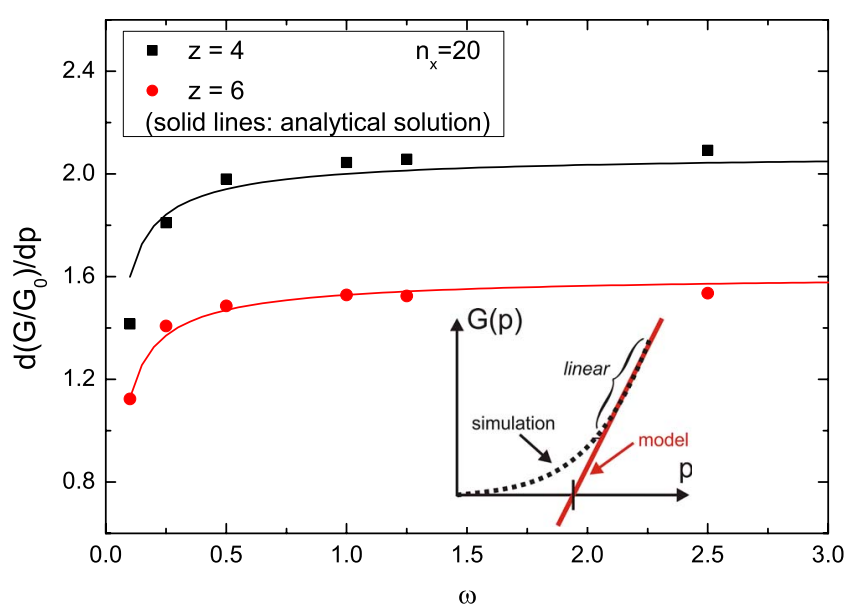

FIG. 4. (Color online) Comparison of numerical (points) and analytical results (solid lines) for the slope $\tilde{m}=d\left(G / G_{0}\right) / d p$ for the square and the triangular lattice as a function of the aspect ratio $\omega\left(n_{x}=20\right)$.

fine $\widetilde{m}$ as the slope of $d\left(G / G_{0}\right) / d p$ far from $\widetilde{p}_{c}$ and replace $p_{c}^{\infty}$ in Eq. (6) by the corresponding slope $m^{\infty}$. Isotropic scaling of the system size still strongly affects the absolute value for conduction, so we have to add a prefactor $G_{0}$, which is the conduction of the undiluted system at $p=1$. This gives for the slopes

$$
\frac{\partial\left(G / G_{0}\right)}{\partial p}=\widetilde{m}=m_{\infty}+\Lambda(\omega) n_{y}^{-1 / \nu} .
$$

The results of Monte Carlo simulations for $n_{x}=20$ and the model are compared in Fig. 4 and show reasonable agreement within the given accuracy. The values for the isotropic case $\omega=1$ correspond approximately to the theoretical values of the effective medium approximation for the square system as expected.

Averaged over three different system sizes $n_{x}=10,20$, and 50 , the relative deviations between Monte Carlo simulations and the model are found as $2.4 \% \pm 2.3 \%$ for $z=4$ and $2.6 \% \pm 2.6 \%$ for $z=6$. This still lies within the uncertainty range given by the variation in the constant $c$ of the scaling function. Due to the small system sizes, which have been used $\left(n_{x} \leq 50\right)$ and the increasing influence of the boundaries into the short direction, the errors are more pronounced for decreasing aspect ratios. Similar deviations between simulations and model were already observed for the apparent percolation thresholds. The approximation for the slopes hence provides a similar accuracy as the analog model for $p_{c}$. The most significant changes in slope occur for values of $\omega n_{x}$ approximately below 20 . Above, only slight changes are observed. Note that in the limit of $n_{x}=1$ the system becomes a one-dimensional system and a transition in exponents is expected to occur [24].

For the representation of the slope, two generic definitions can be used, which result in substantially different characteristics. This is especially of interest in physical systems where the characterization or measurement method plays an important role. By replacing the normalized conduction by the absolute conduction $G(p)$, a dependency on the conduction of

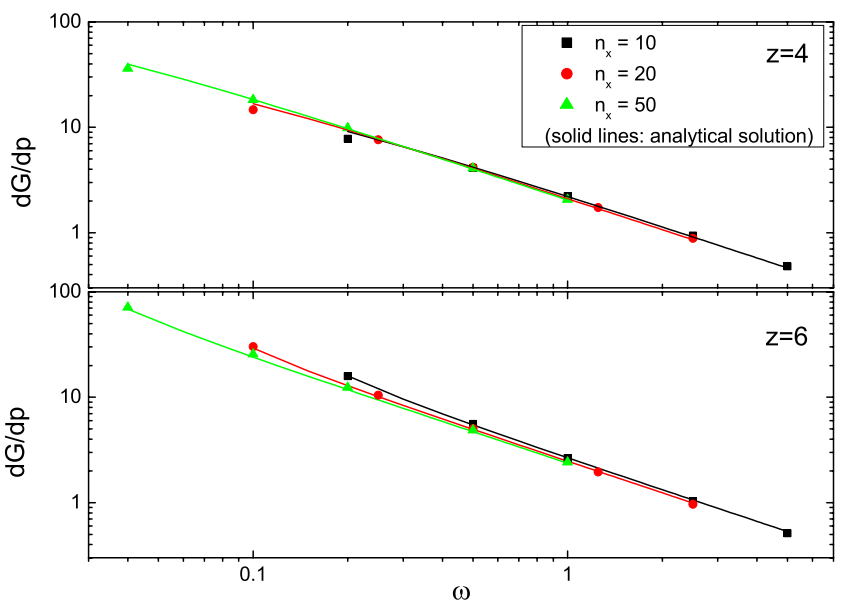

FIG. 5. (Color online) Changed characteristics in aspect ratio dependance of the slope by using absolute conduction $d G / d p$. It is mainly influenced by the conduction of the undiluted lattice $G_{0}$ (points: simulation data; solid lines: analytical solution).

the undiluted lattice $G_{0}$ is added (which in turn is a function of the aspect ratio). Unlike for normalized conduction, the absolute conduction shows decreasing slopes for increasing aspect ratios and steeper slopes for $z=6$ (see results for various system sizes and coordination numbers in Fig. 5).

A general comparison of the topologies shows that the linear chains exhibit a fundamentally different behavior as compared to the two-dimensional systems. While in two dimensions the conduction far away from $p_{c}$ can be well approximated by a linear function, the quasi-one-dimensional case is inherently nonlinear. The change of $\tilde{m}$ with the aspect ratio in $2 \mathrm{D}$ is rather weak. At small values for $\omega n_{x}$ in the regime approximately below 20 , the slope of the conduction increases significantly for all coordination numbers. It is followed by a slow increase with a further increasing aspect ratio.

\section{VALIDITY OF THE LINEAR APPROXIMATION MODEL IN TWO DIMENSIONS}

The linear approximation of the conduction $G(p)$ is only valid afar from the critical region, where the conduction is not anymore described by power laws. To give an estimate of this region for a rectangular system we simply calculate the relative deviations between simulation and linear model $\left[G(p)-G_{\operatorname{lin}}(p)\right] / G(p)$ for varying aspect ratio and system size $n_{x}$, respectively. The results are shown in Fig. 6 for the case of a square $(z=4)$ lattice. The plots end where the linear approximations intersect the $p$ axis. The main graph shows the dependence of the deviations on the systems size for $\omega$ $=0.5$. For high values of $p$ (approximately $>0.75$ ) the relative deviations are close to 0 . For $p$ values approaching the critical region starting from $p=1$ the deviations increase gradually into the positive direction for small system sizes. For increasing system sizes an increasing undershoot into the negative direction is observed, which relaxes toward the intersection point of the approximation with the $p$ axis. It has to be mentioned that the deviation occurs even before the 


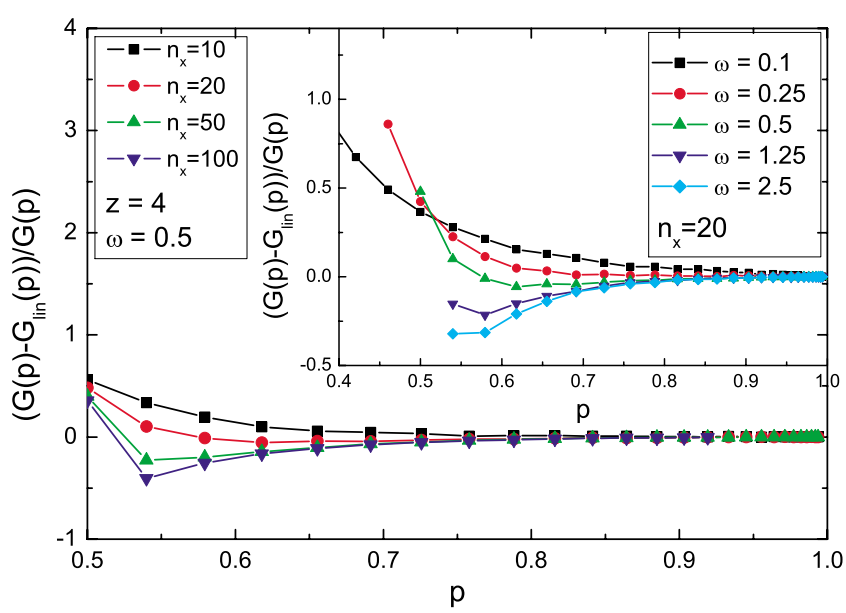

FIG. 6. (Color online) Influence of the aspect ratio $\omega$ and system size $n_{x}$ on the relative deviations of the linear model to conduction predicted by the simulation.

transition region where $P=1$; it is hence not an effect of the finite size of the system only.

Similar characteristics as for changes in system size are obtained when the aspect ratio is changed for a given transversal number of bonds $n_{x}$ (see inset of Fig. 6). For various aspect ratios $\omega$, the predicted slopes deviate at different $p$ values from the simulation. Apart from finite-size effects, this is mainly a result of the increasing percolation thresholds and slopes of the conduction, respectively.

It has to be noted that relatively small systems build the numerical basis for the simulations and the model. Deviations can thus be partly attributed to related effects, making it difficult to quantitatively conclude these results at the present point.

The topology has a great influence on the validity of the linear range as it is seen in Fig. 7 for a system with $n_{x}=20$. The case $z=6$ yields a significantly increased linear range as compared to the case where $z=4$. In the transition region, similar characteristics are obtained in this case as for the

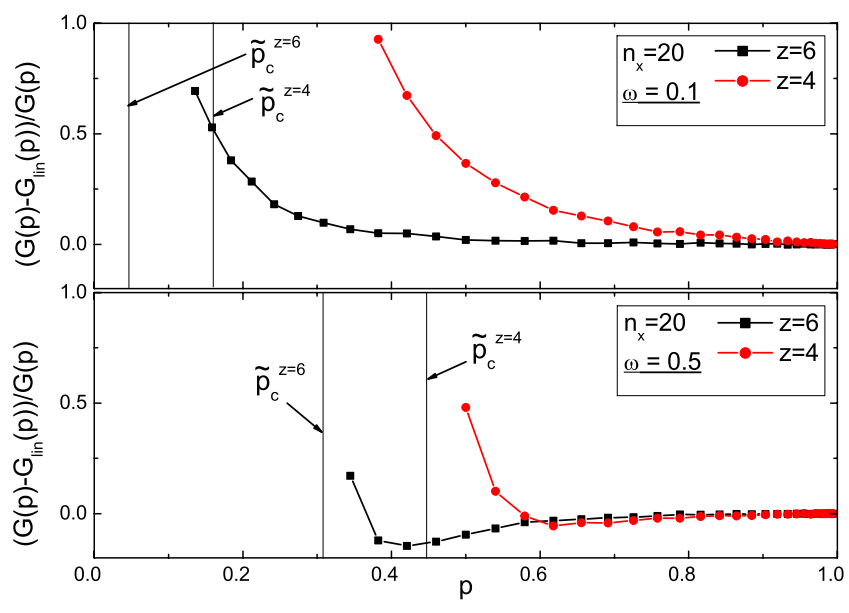

FIG. 7. (Color online) Influence of the coordination number $z$ and aspect ratio $\omega$ on the relative deviations of the linear model to the simulated currents $G(p)$. The largest linear ranges are found for the lower aspect ratios and the high coordination number. square lattice in Fig. 6. Note that for $z=6$ the linear range may exceed the boundaries, which are given by the linear range of the statistical distribution. As already pointed out, the increasing slope for increasing aspect ratios results likewise in a decreased linear range. The quantitative difference in linear range cannot be correlated with the difference in apparent percolation thresholds only, and needs further investigation.

\section{INFLUENCE OF PHYSICAL QUANTITIES}

As it has been introduced, our goal is to relate our results to applications such as electrical transport in percolation based sensors. In such systems, the dependence of the conduction on an externally measurable quantity is of higher interest than on the fraction of occupied bonds itself. This could for instance be the presence of a gas causing a morphological change in a metallic nanostructure [5]. The connection between $p$ and such a quantity, in the following referred to as $\varepsilon$, is given by a specific statistical distribution $f$ (e.g., Gaussian, log-normal), which depends on the physical nature of the system. It reflects the distribution in threshold values $\varepsilon_{t h}$ above which a bond is occupied or not, though practically it may be difficult to quantify in a given situation. The bond distribution $p(\varepsilon)$ is expressed by the corresponding cumulative distribution function (CDF). Both contributions $G(p)$ and $p(\varepsilon)$ to the final conduction can be treated independently. To give a simple example for such a situation we will consider a Gaussian distribution with $f\left(\mu_{\varepsilon}, \sigma_{\varepsilon}\right)$, though others such as the log-normal distribution are widely encountered as well [28].

In this case, the conduction for the quasi-one-dimensional case is readily given over the entire range of $p$ by Eq. (4),

$$
G(\varepsilon)_{1 D}=\left\{\int_{-\infty}^{\varepsilon} \frac{1}{\sqrt{2 \pi} \sigma_{\varepsilon}} \exp \left[-\frac{\left(x-\mu_{\varepsilon}\right)^{2}}{2 \sigma_{\varepsilon}^{2}}\right] d x\right\}^{n_{y}} \frac{n_{x}}{n_{y}} .
$$

In the 2D cases, $G(\varepsilon)$ can be calculated using Eq. (7) and reads as

$$
\begin{aligned}
G(\varepsilon)_{2 D}= & {\left[m^{\infty}+c\left(\omega^{1 / \nu}-1\right) n_{y}^{-1 / \nu}\right.} \\
& \left.\times\left\{\int_{-\infty}^{\varepsilon} \frac{1}{\sqrt{2 \pi} \sigma_{\varepsilon}} \exp \left[-\frac{\left(x-\mu_{\varepsilon}\right)^{2}}{2 \sigma_{\varepsilon}^{2}}\right] d x-1\right\}\right] G_{0},
\end{aligned}
$$

where $G_{0}$ is again the conduction of the undiluted lattice. Note that the dependence of $G$ on the parameter $\varepsilon$ changes the range of linearity of $G(\varepsilon)$ as compared to $G(p)$. While $G(p)$ has shown to approximately follow a linear function for $p \rightarrow 1$, this is no longer the case for $G(\varepsilon)$, caused by the nonlinearity of the CDF. The emerging limits can be approximated by linearizing $G(\varepsilon)$ around a working point on the CDF. In case of the Gaussian distribution we choose the slope at $\mu_{\varepsilon}$ for symmetry reasons and obtain as the linear approximation at this point, 


$$
p_{\text {lin }}(\varepsilon)=\frac{1}{2}+\frac{\varepsilon-\mu_{\varepsilon}}{\sqrt{2 \pi} \sigma_{\varepsilon}} .
$$

We now define a maximum relative deviation $\delta$ between the linear approximation whereby the original function and the upper limit $\varepsilon_{u}$ for linearity can be readily calculated. The corresponding value of $p_{u}$ is obtained by evaluating the CDF at $\varepsilon_{u}$. With an arbitrarily chosen value of $\delta<5 \%$ and numerical evaluation of the resulting equation $G\left(\varepsilon_{u}\right)<1.05 p_{\operatorname{lin}}\left(\varepsilon_{u}\right)$, we obtain an upper limit of $p_{u} \approx 0.85$, which is independent of the parameters $\mu_{\varepsilon}$ and $\sigma_{\varepsilon}$ of the Gaussian distribution function. A shift of both in $\mu_{\varepsilon}$ and $\sigma_{\varepsilon}$, respectively, solely causes a linear shift in $\varepsilon_{u}$. In analogy, a lower limit of linearity $p_{l}$ can be defined. For the given parameters and solving the equation $G\left(\varepsilon_{l}\right)<0.95 p_{\text {lin }}\left(\varepsilon_{l}\right)$ we obtain $p_{l} \approx 0.26$.

Using this approximation with its boundaries, the slope $\tilde{m}^{*}$ of the conduction $G(\varepsilon)$ in dependence of the parameter $\varepsilon$ is obtained as

$$
\tilde{m}_{2 D}^{*}=\frac{\partial p}{\partial \varepsilon} \frac{\partial G}{\partial p}=\frac{m^{\infty}+c\left(\omega^{1 / \nu}-1\right) n_{y}^{-1 / \nu}}{\sqrt{2 \pi} \sigma_{\varepsilon}},
$$

which is only dependent on the width $\sigma_{\varepsilon}$ of the distribution.

\section{CONCLUSIONS}

In this work we studied conduction in finite rectangular random resistor networks in two dimensions, motivated by its usability for nanostructured materials and sensing appli- cations. We showed that the same scaling function, which has been introduced by Masihi et al. [24] for apparent percolation thresholds also applies for a linear approximation of the conduction far away from the percolation threshold in two dimensions. We performed Monte Carlo simulations on lattices with moderate geometrical aspect ratios and various topologies such as parallel linear chains, square, and triangular lattices. Given the statistical errors of the simulations and the accuracy of the numerical constants within the model, a reasonable approximation for the slope of the conduction far away from the percolation threshold is obtained.

We find that for equivalent geometries the triangular lattice shows lower percolation thresholds, flatter slopes of the conduction and a larger range where this approximation is legitimate. Parallel linear chains present a fundamentally different behavior due to their quasi-one-dimensional nature, with pronounced shifts in percolation thresholds and conduction over a wide range. This topology shows the narrowest linear range but the steepest obtainable slopes.

We further linked the results to physical quantities, which are described by a distinct statistical distribution and influence the fraction of conducting bonds. These results provide useful information for the development and the analysis of percolation based functional materials or sensors.

\section{ACKNOWLEDGMENTS}

The authors thank Frédéric Favier and Ricardo Pérez Suárez for helpful discussions and comments.
[1] J. R. Morris and T. J. Coutts, Thin Solid Films 47, 3 (1977).

[2] K. Sieradzki, K. Bailey, and T. L. Alford, Appl. Phys. Lett. 79, 3401 (2001).

[3] A. G. Hunt, Adv. Water Resour. 24, 279 (2001).

[4] D. Zhang, M. A. Kandadai, J. Cech, S. Roth, and S. A. Curran, J. Phys. Chem. B 110, 12910 (2006).

[5] O. Dankert and A. Pundt, Appl. Phys. Lett. 81, 1618 (2002).

[6] E. C. Walter, F. Favier, and R. M. Penner, Anal. Chem. 74, 1546 (2002).

[7] S. Kirkpatrick, Rev. Mod. Phys. 45, 574 (1973).

[8] D. Stauffer, Phys. Rep. 54, 1 (1979).

[9] D. Stauffer and A. Aharony, Introduction to Percolation Theory, 2nd ed. (Taylor \& Francis, London, 2003),.

[10] B. Berkowitz and R. P. Ewing, Surv. Geophys. 19, 23 (1998).

[11] S. Havlin and D. Ben-Avraham, Adv. Phys. 36, 695 (1987).

[12] R. W. Rendell, M. G. Ancona, W. Kruppa, E. E. Foos, A. W. Snow, D. Park, and J. B. Boss, IEEE Trans. Nanotechnol. 2, 75 (2003).

[13] E. Z. Meilikhov, Phys. Lett. A 346, 193 (2005).

[14] M. Sahimi, B. D. Hughes, L. E. Scriven, and H. T. Davis, J. Phys. C: Solid State Phys. 16, L521 (1983).

[15] J. Bernasconi, Phys. Rev. B 18, 2185 (1978).
[16] M. Sahimi, B. D. Hughes, L. E. Scriven, and H. T. Davis, Phys. Rev. B 28, 307 (1983).

[17] J.-P. Hovi and A. Aharony, Phys. Rev. E 56, 172 (1997).

[18] P. Grassberger, Physica A 262, 251 (1999).

[19] P. Lajkó and L. Turban, J. Phys. A 33, 1683 (2000).

[20] R. A. Monetti and E. V. Albano, Z. Phys. B: Condens. Matter 82, 129 (1991).

[21] R. A. Monetti and E. V. Albano, Z. Phys. B: Condens. Matter 90, 351 (1993).

[22] R. A. Monetti and E. V. Albano, J. Phys. A 26, 3955 (1993).

[23] S. Tsubakihara, Phys. Rev. E 62, 8811 (2000).

[24] M. Masihi, P. R. King, and P. Nurafza, Phys. Rev. E 74, 042102 (2006).

[25] F. Favier, E. C. Walter, M. P. Zach, T. Benter, and R. M. Penner, Science 293, 2227 (2001).

[26] C. Pennetta, E. Alfinito, L. Reggiani, F. Fantini, I. DeMunari, and A. Scorzoni, Phys. Rev. B 70, 174305 (2004).

[27] J. S. Andrade, Jr., Y. Shibusa, Y. Arai, and A. F. Siqueira, Synth. Met. 68, 167 (1995).

[28] C. G. Granqvist and R. A. Buhrman, J. Appl. Phys. 47, 2200 (1976). 